\title{
Neonatal deaths in Cambodia: a case series
}

\author{
A.N. Bazzano ${ }^{1}$, C. Var $^{2,3}$, D. Wilkosz ${ }^{1}$, R. Duggal ${ }^{4}$, R.A. Oberhelman ${ }^{1}$
}

${ }^{1}$ Department of Global Community Health and Behavioral Sciences, Tulane University School of Public Health and Tropical Medicine, 1440 Canal St., New Orleans, LA 70112, USA

${ }^{2}$ National Institute of Public Health, \#2 Kim Y Sung Blvd, Tuol Kork, P.O. Box 1300, Phnom Penh, Cambodia

${ }^{3}$ Reproductive Health Association of Cambodia (RHAC), \#5, 150 St., Phnom Penh, Cambodia

${ }^{4}$ Tulane University School of Medicine, 1430 Tulane Ave, New Orleans, LA, 70112 USA

Keywords Infant, newborn; Cambodia; child mortality; perinatal mortality; case reports

\begin{abstract}
Introduction: Neonatal mortality has declined in Cambodia but remains a key contributor to under-five deaths. The aim of this study was to further understanding of potential factors contributing to high neonatal mortality rates in Cambodia through assessment of verbal autopsies collected following newborn deaths. The study team analyzed verbal autopsies of perinatal deaths in order to describe timing and causes of neonatal deaths, demographic data, and factors potentially related to mortality. Methods: The case series data derive from 13 verbal autopsy reports collected in rural southern Cambodia. The mortality review was nested within a trial of a behavioral intervention to improve newborn survival, and was conducted after the close of the trial. The study examined all neonatal deaths occurring to infants born at 16 health centers between in the study site of Takeo province. The World Health Organization standardized definition of neonatal mortality was employed, and two pediatricians independently reviewed data collected from each event to assign a cause of death. Results: Thirteen newborn deaths of infants born at a health facility were reported during the time period February 2015 - November 2016. Ten out of the 13 deaths $(76.92 \%)$ were early neonatal deaths, two $(15.38 \%)$ were late neonatal deaths, and one was a stillbirth. Five out of 13 deaths $(38.46 \%)$ occurred within the first day of life, indicating death was likely due to an intrapartum event. The largest single contributor to mortality was neonatal sepsis; six of 13 deaths $(46.15 \%)$ were attributed to some form of sepsis. Twenty-three percent of the deaths were attributed to asphyxia. Other causes of death included stillbirth and prematurity. Eight deaths $(61.54 \%)$ occurred within the control group of the larger intervention study. Conclusion: The study highlights the continuing need to improve both intrapartum and postnatal quality of care and infection prevention and control, and to fully address causes of sepsis, in order to effectively reduce mortality in the newborn period.
\end{abstract}




\section{Background}

The first 28 days of life are critical for a child's survival, when infants face the highest risk of mortality. In 2016, 46\% of under-five deaths occurred during the neonatal period.[1] The vast majority of newborn deaths occur in low-income countries where access to health care is restricted. In Cambodia, neonatal mortality rates have dropped considerably in recent years, from 24 neonatal deaths per 1,000 live births in 2009 to 18 per 1,000 live births in 2014; however, regionally, the newborn mortality rate varies significantly, from a high of 36 neonatal deaths per 1,000 live births in the Mondul Kiri and Ratank Kiri provinces, to a low of 12 per 1,000 live births in Battambang and Pailin provinces.[2] The national rate is still nearly three times higher than the World Health Organization Western Pacific regional average of 6.5, and a child born in Cambodia is still significantly more likely to die than one born in a high-income country.[1]

In 2009, the Cambodia Ministry of Health developed a fast-track initiative in an effort to address maternal and child health outcomes, however, the agenda lacked specific strategies to prevent neonatal deaths in the postnatal period. Formative research found gaps in essential newborn care practices [3] and barriers to infection prevention and control.[4] The data from that formative research guided an intervention design for a clustered randomized trial to assess the impact of a package intervention on newborn health in the Takeo province of southern Cambodia, the Newborn Infection Control and Care Initiative (NICCI) trial [5]. The trial aimed to improve care practices and care-seeking for newborns at the health center, community, and household levels. Study objectives included improving infection control and referral systems in selected health centers, increasing knowledge and recognition of danger signs for sick newborns by mothers and families of newborn infants, and diagnosing the causes of sepsis among infants with possible sepsis at Takeo Province Hospital.

Following the end of the intervention, which took place over the course of 22 months from 2014-2016, data on 13 neonatal deaths had been collected. Researchers conducted verbal autopsy (VA) interviews using a standardized questionnaire with family members of newborns to ascertain cause of death. Per the World Health Organization (WHO), "Verbal autopsy is a method used to ascertain the cause of a death based on an interview with next of kin or other caregivers. This is done using a standardized questionnaire that elicits information on signs, symptoms, medical history and circumstances preceding death. The cause of death, or the sequence of causes that led to death, are assigned based on the data collected by a questionnaire and any other available information." These interviews are frequently used in settings where the majority of deaths occur at home or in resource-poor areas where there is little chance that deaths will be recorded and causes of death determined.[6]

The current study aimed to describe the factors potentially contributing to these 13 neonatal deaths in southern Cambodia. Specifically, to analyze the verbal autopsies of perinatal deaths in both intervention and control groups, describe the timing and causes of these deaths, describe the basic demographics of newborns and mothers, and to understand the factors surrounding these deaths. 


\section{Methods}

The case series examining neonatal deaths was conducted through a retrospective review of 13 verbal autopsy reports. The standardized definition from the WHO for neonatal mortality was used as the inclusion criteria for this study. Two pediatricians reviewed verbal autopsy data and assigned a presumed cause of death, per WHO verbal autopsy guidelines.[6] Neonatal death was defined as death of a live-born child occurring within 28 days of life. Early neonatal death referred to death occurring within seven days of life while death occurring on or after seven days but before 28 days was referred to as late neonatal death.[7] Additionally, the WHO definition of intrapartum and very early neonatal mortality — occurring within the first 24 hours of life and excluding newborns under 2,500 grams - was used to identify births that occurred due to intrapartum events.[8]

\section{Setting}

Takeo province is located in southern Cambodia, bordering Vietnam. The population, as of 2013, was 923,373.[9] Cambodian provinces are subdivided into administrative districts and communes, and Takeo has 10 districts and 100 communes. The country's health system includes minimum care health centers and posts, mainly for rural populations, and these facilities provide referrals to district, provincial, and national hospitals. There are eight national hospitals and in Takeo, there are 73 health centers, three primary referral hospitals, and one secondary referral hospital.[10]

\section{Data collection and analysis}

Verbal autopsy questionnaires were administered in Khmer by trained members of the larger intervention study, during the process of monitoring newborn deaths in the study area, and who had post secondary education in midwifery. The questionnaire had both open and closed-ended questions and included portions that provided for respondent's verbatim account of the circumstances leading to the death of the child. Immediate caregivers (most often mothers) were the primary target respondents. The interviews were conducted on average of 2 months after death had occurred. Interviews lasted between 30 minutesand one hour and informed consent was obtained from all participants. The study was approved by the National Ethics Committee Health Research of the Cambodia Ministry of Health and by the Institutional Review Board of Tulane University. The parent study was registered with ClinicalTrials.gov, number NCT02271737. Descriptive data were extracted from 13 verbal autopsy forms reporting on deaths between February 2015 and November 2016. Analysis included summary statistics of causes and timing of deaths, as well as a qualitative description of circumstances surrounding the birth and death.

\section{Results}

Case 1 was a female born at 33 weeks' gestation, weighing 3,300 grams. Her healthy 22 year-old mother delivered at a local health facility; labor lasted seven hours and the birth was breech. Her mother reported the baby cried, moved, and breathed post-delivery and nursed normally; she was breast and formula-fed. Mother and baby left within one hour of delivery. On day of life (DOL) 2, mother noticed bleeding at the baby's vaccine site; the verbal autopsy report also showed that the baby was vomiting all feeds and was jaundiced. The mother took the baby to a local government clinic where she was referred to a hospital. The baby died two days later in the hospital where she was taken; cause of death was determined to be other and unspecified perinatal cause of death, possibly due to dehydration.

Case 2 was a boy born at 40 weeks' gestation, weighing 3,600 grams. His previously healthy 28 year-old mother reported several health issues during her third trimester, including severe anemia and shortness of breath; fast, difficult breathing; and having a fever with vaginal discharge and abdominal pain, however, 
she did not seek care for these symptoms. His mother went to a local clinic six hours after her water broke and was in labor for 24 hours. The verbal autopsy indicated green, foul-smelling amniotic fluid and that the baby was born with bruising and did not breathe in the first five minutes post-birth. Baby was never able to suckle normally and was given breast milk but not breastfed; in the verbal autopsy his mother reported the baby was lethargic, had difficulty breathing, blackened skin, and did not cry. The baby was referred to Takeo Hospital post-delivery, due to the facility's inability to manage his symptoms, but he died before leaving the facility on DOL 1; cause of death was determined to be asphyxia.

Case 3 was a premature male born at 30 weeks' gestation. He weighed 2,200 grams and was born breech; he died at two days old. His 32 year-old mother reported that while the baby cried and breathed immediately after birth, he had difficulty breathing, fast breath, and was never able to suckle and breastfeed normally. She also reported that on DOL 1, the baby was grunting and became cold to the touch. The baby did not receive any care for these symptoms and mother and baby left their local health facility 10 hours after delivery; while the baby was examined by a health worker prior to being discharged, his mother did not receiving any type of counselling. On DOL 2, the mother reported the baby's skin began to turn black and she sought care seven hours later at the local hospital, where the baby was given oxygen and a breathing bag or mask, in addition to fluids by mouth and IV. The baby died on DOL 3 and cause of death was determined to be prematurity.

Case 4 was a boy born at 37 weeks' gestation, weighing 3,500 grams. His grandmother, who was present during pregnancy and delivery and gave the interview for the verbal autopsy, reported that his healthy mother gave birth at a local health facility and that the labor lasted 24 hours. She also reported that while the baby breathed immediately after birth, he had difficulty breathing and did not cry or suckle normally. Mother and baby left the facility one hour after birth. The grandmother reported that the baby was choking on amniotic fluid because of prolonged labor and was eventually taken to a government health center for these symptoms, though she was unsure how long that occurred after symptoms set in. From there, the baby was referred to a local hospital where he was admitted and died at four days old.

Case 5 was a boy born late preterm at 36 weeks' gestation. He weighed 3,500 grams and died at one day old. Like Case 4, the baby's grandmother gave the interview for the verbal autopsy and was present during the mother's pregnancy and delivery. She reported that the baby's mother had anemia and shortness of breath, vaginal bleeding, and foul-smelling discharge in her last trimester and before labor; during her pregnancy she sought care for these symptoms at the hospital and was able to follow the advice given to her by her provider. The mother's delivery occurred at a local health provider and lasted five hours. The baby's grandmother reported that while he did breathe immediately after birth, he did not cry and was never able to suckle normally. On DOL 1 , the baby had difficulty breathing, fast breath, became lethargic, and had redness and swelling of the skin, which then turned black. The mother and baby were referred to a local hospital upon discharge — one hour after birth — where the baby was given oxygen, a breathing bag/mask, and fluids by mouth and IV. He died that same day and cause of death was determined to be neonatal sepsis.

Case 6 was a girl born at 38 weeks, weighing 4,400 grams. Her previously healthy 35 year-old mother reported fast breathing and trouble sleeping during her last trimester, for which she sought treatment at a government clinic. The mother went to a local health facility for delivery and labor lasted for five hours. The mother reported green, foul-smelling amniotic fluid, as well as bruises on the baby's body at birth. The mother also reported that the baby did not immediately breathe after birth, and though she did cry immediately after birth, stopped being able to cry. The baby was able to suckle normally and was given powdered formula. Fast and difficult breathing were reported having started on DOL 1 and lasted up until the baby's death, at 12 days old; the baby also had a fever, became unconscious, had a bulging fontanelle, 
and areas of the skin that turned black in the illness leading up to her death. The baby was referred to a local hospital upon discharge, seven hours after birth. Upon arrival she was given oxygen, a breathing $\mathrm{bag} / \mathrm{mask}$, and fluids by mouth and IV. She died 12 days later and the cause of her death was determined to be neonatal sepsis, possibly meningitis.

Case 7 was a male born at 39 weeks, weighing 3,500 grams; he died the day he was born. His grandmother gave the interview and like Case 2 and Case 4, reported being present for the mother's pregnancy and delivery. Delivery occurred at a health facility and lasted for three hours. The grandmother reported green/brown amniotic fluid and that post-delivery, an antibiotic ointment was applied to umbilical cord stump. Immediately after birth, the baby had difficulty breathing and stopped being able to cry; he was formula-fed, though the grandmother did not know whether he was ever able to suckle. During the illness that led to death, the verbal autopsy report indicates the baby was grunting, cold to the touch, became lethargic, and had redness of the umbilical cord stump. The grandmother also reported that the baby had yellow eyes and appeared to be healthy and then died suddenly, one hour after birth, never leaving the facility in which he was born. Cause of death was omphalitis.

Case 8 was a male born at 40 weeks old; he weighed 3,000 grams and was our study's only stillbirth. His mother's brother gave the interview for the verbal autopsy, and reported being present for mom's pregnancy and delivery. The mother first attempted to go to a health center after labor began but ended up at a private clinic seven hours later because the provider was not able to manage her delivery. Although, according to her brother, the mother felt the baby move one hour before delivery, labor and delivery took three days before a cesarean section was eventually performed; the baby had bruising and microcephely. The mother remained at the private clinic for two days post-delivery.

Case 9 was a boy born at 42 weeks' gestation, weighing 2,600 grams. His previously healthy 21 year-old mother reported having fastness of breath in her last trimester, for which she did not seek treatment. She also reported having fast or difficult breathing during labor and delivery, and she first sought care at a local health center one hour after labor started. The baby was breech and so, four hours later she was referred to the local hospital because the provider was unable to deliver the baby; there she was given medicine to strengthen the labor and the baby was delivered vaginally with forceps. The mother reported the baby had difficulty breathing and did not cry immediately after birth. The baby was formula fed and never able to suckle normally. Starting on DOL 1 and leading up to his death, the baby had difficulty breathing, fast breathing, indrawing of the chest, grunting, and fever. He was given oxygen and a breathing bag/mask but died at 3 days old. Cause of death was neonatal pneumonia.

Case 10 was a female, born at 40 weeks, weighing 3,300 grams. Her 29 year-old mother reported having an inflamed bowel and UTI prior to her pregnancy and reported severe abdominal pain and smelly vaginal discharge in her last trimester. She sought care for these symptoms at a private clinic and was told to rest and return for a follow-up visit, which she did. She went to a local health center two hours after labor started and reported having high blood pressure during labor and delivery. Seven hours later, she was referred to a hospital because the provider was unable to manage the problem. There she was given medicine to strengthen labor and IV fluids and the baby was born with no immediate signs of illness- he cried, moved, breathed, and nursed normally initially. His mother reported the blackening of skin but that he appeared healthy and then died in the hospital soon after birth, the day after he was born. Cause of death was determined to be other and unspecified.

Case 11 was a boy born at 37 weeks' gestation, weighing 4,000 grams. His otherwise healthy 22 year-old mother sought care from a private clinic during pregnancy for severe headaches; she was given medication and ordered to rest and drink warm water. She reported not taking the medication because she 
was worried it would harm her unborn child. Her water broke more than six hours before labor began, and during her 13-hour labor she had severe abdominal pain and vaginal bleeding; she also reported green/brown amniotic fluid and was given IV fluids. The baby was born with bruising and it took more than 30 minutes for the baby to breathe or cry after birth; he was never breastfed and stopped being able to suck normally on DOL 2. His mother reports that during the illness that led to death, he had difficult and fast breathing, a fever, became lethargic, became unconscious, and had blackened skin. Mom and baby left the facility one hour after birth, and were referred to Takeo hospital for treatment. Fifteen hours later, the baby was referred to Kuntheak Bopha hospital — three hours away — because Takeo hospital could not manage the problem. The baby died at Kuntheak Bopha at two days old; cause of his death was birth asphyxia.

Case 12 was a male born at 43 weeks gestation, weighing 3,800 grams; he died at 11 days old. His previously healthy 19 year-old mother reported having vaginal bleeding and foul-smelling discharge in her third trimester, for which she did not seek treatment. She went to a local health center when labor started, and labor and delivery took six hours. The mother reported seeing bruises on the baby's body at birth, but he appeared to be otherwise healthy - he was exclusively breastfed and able to suckle normally and mom and baby left the health facility one hour after birth. Eight days later, his mother noticed redness and bleeding at the site of the umbilical cord stump; she immediately took him to a local health center and they were then referred to hospital as the health center could not manage the problem. He was admitted to the hospital and given oxygen, however, he died three days later-cause of death was determined to be omphalitis.

Case 13 was a boy born at 44 weeks; he weighed 3,500 grams. His previously healthy 28 year-old mother reported having high blood pressure and severe abdominal pain in her last trimester, for which she sought treatment and was given blood pressure pills. She gave birth at a health facility and labor and delivery lasted for three hours. The baby did not cry or breathe immediately after birth and required intervention. He was breastfed up until illness set in, and was then switched to formula. On DOL 1, he was having difficulty breathing and was grunting. He left the facility eight hours after birth, but on DOL 2, fever and jaundice set in, the baby became lethargic, and areas of the skin turned black. He was taken to a hospital two hours away, where he was given oxygen, a breathing bag/mask, fluids and other oral medication. He remained there for one day, where he died at age two days; cause of death was determined to be neonatal sepsis.

Thirteen newborn deaths occurred. Eight of those deaths (61.54\%) occurred within the control group. Ten out of all 13 deaths (76.92\%) were early neonatal deaths, two (15.38\%) were late neonatal deaths, and one was a stillbirth. The largest single contributor to neonatal death in the sample, per cause of death assigned by consulting pediatricians, was neonatal sepsis. Six out of 13 deaths $(46.15 \%)$ were attributed to some form of sepsis. Twenty-three percent of the deaths were attributed to asphyxia. Other causes of death included stillbirth and prematurity.

The majority of the newborns (76.92\%) were male, and their mothers' ages ranged from 19 to 35 , whom were married between the ages of 17 and 27 . The mothers had an average of 6.7 years of schooling and their average household size was 6.4 people per home.

The number of mothers' antenatal care visits ranged from two to nine, with an average of five. At those visits, only six women were informed of any danger signs to be aware of during pregnancy or where to go if she was experiencing any of those signs.

All 13 newborns were born in a health facility, and five (38.46\%) died at the same facility in which they were born. Of the eight babies who went home after birth, four (50\%) were referred to a hospital upon 
discharge of the facility where they were born; all four died in the hospital after referral. Of those mother/baby pairs who went home after birth, none of them reported being visited by a community health worker at home.

Twelve out of 13 (92.31\%) deliveries were vaginal, with the exception being the stillbirth, where cesarean section was used to deliver the baby. Of the 12 vaginal deliveries, three $(25 \%)$ were with forceps. Four of the 13 babies (23.08\%) were ever breastfed, and seven of $13(53.85 \%)$ were fed either a pre-mixed formula or powdered formula mixed with a liquid. Three out of 13 mothers $(23.08 \%)$ received counselling upon discharge of the birth facility that did not include referral to a hospital; two of those three were in the intervention group.

\section{Early neonatal deaths}

Ten out of the 13 recorded deaths (76.92\%) were within the first week of life, five of which $(38.46 \%)$ occurred between zero and one day. Four out of the 10 early neonatal deaths $(40 \%)$ were within the intervention group. The largest contributors to early neonatal death in our sample were neonatal sepsis and asphyxia. Three of the 10 early neonatal deaths (30\%) were due to neonatal sepsis and three more of the $10(30 \%)$ were due to asphyxia. Other causes of early neonatal death recorded were other/unspecified, pneumonia, and prematurity.

\section{Late neonatal deaths}

Two of the 13 recorded deaths $(15.38 \%)$ occurred between the $7^{\text {th }}$ and $28^{\text {th }}$ day of life - one from the control group and one from the intervention group. Neonatal sepsis was the determined cause of death in both cases.

\section{Discussion}

The purpose of this case series was to further understand circumstances related to neonatal mortality in rural Cambodia, within the context of a trial that aimed to improve care practices and care-seeking for newborns at both the health center and community level. We report on the causes of deaths of 13 neonates following verbal autopsy interviews with family members of the deceased. Five of the deaths reported $(38.46 \%)$ were linked to intrapartum events, including four of the five deaths that occurred within the intervention group. Severe infection was the most common cause of death, followed by asphyxia.

Verbal autopsy analyses conducted in other countries indicate that sepsis and asphyxia are often among the top three causes of neonatal deaths. When conducting verbal autopsies in Morang, Nepal, Khanal et al. found that infection (41\%), birth asphyxia (37\%), and prematurity or low birth weight (18.4\%) were the most common causes of neonatal death.[11] An analysis of verbal autopsies in Nigeria found that sepsis $(31.5 \%)$, birth injury/asphyxia (22.3\%), and pneumonia (19.9\%) were the largest contributors to death.[12] A VASA investigation conducted by the World Health Organization and UNICEF to estimate the causes of neonatal and child deaths in several high priority countries found that severe neonatal infection and asphyxia were the leading causes of neonatal death in Niger in 2010.[13]

While neonatal sepsis and asphyxia were found to be the leading causes of neonatal deaths in this series, indicating sub-optimal intrapartum and postnatal care, other studies identified underlying causes more specific to the study setting or sociodemographic characteristics. For example, Kulkarni and colleagues found low birth weight to be the main cause of 52 early neonatal deaths in Maharashtra, India, followed by prematurity. Prematurity was also the most common cause of stillbirth, and was the underlying cause of death in $7.6 \%$ of deaths that were due to low birth weight. One study identified an important linkage to 
mother's age--more than half of recorded stillbirths in that study were to women under 21 years old, and babies born to mothers under 25 years old were less likely to survive compared to older mothers.[14] Research in Bangladesh and Malawi found delays in care-seeking to be an underlying cause of neonatal deaths.[15,16] In the current study, only one case reported pre-term delivery and there was limited information on care-seeking, likely related to the small sample size.

Our findings suggest that, despite the majority of birth attendants having reporting to have followed recommended infection prevention control practices for intrapartum care (as noted in the interview) including washing hands, wearing gloves, and proper cord care - the majority of deaths $(46.15 \%)$ were due to sepsis, indicating infection control remains a challenge. Additionally, prompt initation and exclusive breastfeeding, which are known to protect against neonatal illness including sepsis $[17,18]$ were not widely practiced among cases in this series, with only five families reporting breastfeeding of baby before death.

Additionally, the VA interviews highlight several important gaps regarding referral and postnatal care. First, no respondent who left the facility where she gave birth reported being visited by a community health worker at home, pointing to a lack of care coordination between health center staff and village health volunteers. Second, while seven mothers received some form of counselling upon discharge, four of those women were referred to a hospital, and of the three mothers who received counselling other than a hospital referral, only two received information regarding danger signs of newborn illness. Data regarding the amount of time between recognition of danger signs and seeking care was slightly more promising, however. Of the eight women who were discharged post-delivery, all eight reported seeking care within one day of deciding to take the baby to a health provider.

This study's limitations include the small number of cases identified. Additionally, four of the respondents interviewed were not the mothers of the deceased (but rather were close family members who confirmed being present for pregnancy, delivery, and during the child's illness and death) which may have impacted recall. Finally, some VA records contained very limited narrative detail. Limited information such as this is common in community-based studies, and is to be expected given the sensitive nature and length of verbal autopsy interviews, but nonetheless restricts comprehensive analysis.

\section{Conclusion and Recommendations}

Improving quality of neonatal care is critical to reaching the Sustainable Development Goals [19], particularly in countries where birth in health facilities with skilled attendants is rapidly improving. While neonatal mortality rates have steadily dropped in Cambodia, ensuring quality of care remains a persistent challenge, as evidenced by the Ministry of Health's renewed focus on maternal and newborn health. This case study series sought to explore the continuing challenges related to perinatal survival in Takeo province, within the context of an intervention that aimed to improve care practices, coordination, and the recognition of danger signs of newborn illness. A few key points can be applied to future research and intervention strategies to improve newborn survival.

All of the mothers interviewed in our sample sought care at local health centers, and the majority of newborn deaths were early neonatal deaths. Improving quality of care and health center protocols for managing birth complications at small health facilities must be a priority. For example, being able to identify and assist a newborn suffering birth asphyxia can reduce mortality--asphyxia accounted for $23 \%$ of the deaths in our sample.[20] In addition, nearly half of deaths in this study were due to some form of neonatal sepsis, indicating infection prevention control at the level of the health centers may have been 
inadequate. Sub-optimal infection prevention and control (IPC) practices have been identified in other settings as important contributors to mortality and morbidity.[21]

Secondly, prompt referral to higher levels of care and assistance in overcoming barriers to referral must also be addressed where care providers are not able to manage newborn illness. In Cambodia, newborns delivered at health centers almost always need to be treated for newborn illness at a referral hospital due to lack of training and equipment at the health center level, yet they are very likely to present there at the first instance.

Finally, counseling to initiate breastfeeding immediately following delivery should be a priority focus for midwives caring for new mothers in this setting - however, more than half of the babies in our sample were never breastfed, and upon discharge only three women received any type of counselling, which includes advice on practices such as breastfeeding.

A systematic approach to understanding, identifying, and managing barriers to improving quality of care for neonatal illness is critical to ensuring that Cambodia's trends of lower neonatal mortality rates continues.

\section{Acknowledgements}

We are grateful to the families who participated in this work. We also appreciate Prof. Chhea Chhorvan, Director of National Institute of Public Health; Prof. Tung Rathay, Director of National Maternal and Child Health, and Dr. Nuth Sinat, Director of Pronvical Health Department for their guidance and administrative support. We would like to acknowledge the contribution of the following individuals: Dr. Lean Supheap, NIPH; Dr. Iv Ek Navapol, RHAC; Dr. Te Vantha, Takeo Provincial Hospital; Dr. Seng Bunly, BNC Consult; and Mrs. Uy Chan and Mrs. Long Leakhana (Takeo MCH), for their technical expertise and operation support. The study was funded through a sub-grant award from the National Academy of Sciences (NAS), funded by United States Agency for International Development (USAID) under prime agreement number AID-OAAA-A-11-00012.

\section{Conflict of interest statement}

The authors declare that they have no conflicts of interest to report. 


\section{References}

1. Hug L, Sharrow D, Danzhen Y. Levels \& trends in child mortality, report 2017: Estimates developed by the UN Inter-agency Group for Child Mortality Estimation. United Nations Children Fund; 2017.

2. National Institute of Statistics, Directorate General for HC, International ICF. Cambodia Demographic and Health Survey 2014. Phnom Penh, Cambodia: National Institute of Statistics/Cambodia, Directorate General for Health/Cambodia, and ICF International; 2015.

3. Bazzano AN, Oberhelman RA, Potts KS, Taub LD, Var C. What health service support do families need for optimal breastfeeding? An in-depth exploration of young infant feeding practices in Cambodia. International Journal of Women's Health; 2015;7:249-57.

4. Bazzano AN, Oberhelman RA, Potts KS, Gordon A, Var C. Environmental Factors and WASH Practices in the Perinatal Period in Cambodia: Implications for Newborn Health. International Journal of Environmental Research and Public Health. 2015;12(3):2392-410.

5. Var C, Bazzano AN, Srivastav SK, Welty JC, Ek NI, Oberhelman RA. Newborn Infection Control and Care Initiative for health facilities to accelerate reduction of newborn mortality (NICCI): study protocol for a randomized controlled trial. Trials. 2015;16:257.

6. World Health Organization. Verbal autopsy standards: the 2012 WHO verbal autopsy instrument Release Candidate 1. Geneva: World Health Organization; 2012.

7. World Health Organization. Neonatal and perinatal mortality: Country, regional and global estimates. France: World Health Organization; 2006.

8. World Health Organization. Monitoring emergency obstetric care: A handbook. Geneva: World Health Organization; 2009.

9. National Institute of Statistics, Ministry of Planning. Economic census of Cambodia 2011, provincial report: 21 Takeo province. Phnom, Penh. 2013.

10. Bureau of Health Economics and Financing, Department of Planning and Health Information. Annual health financing report 2012. Phnom Phen. Bureau of Health Economics and Financing, Department of Planning and Health Information; 2013.

11. Khanal S, Gc VS, Dawson P, Houston R. Verbal autopsy to ascertain causes of neonatal deaths in a community setting: a study from Morang, Nepal. JNMA J Nepal Med Assoc. 2011;51(181):217.

12. Adewemimo A, Kalter HD, Perin J, Koffi AK, Quinley J, Black RE. Direct estimates of causespecific mortality fractions and rates of under-five deaths in the northern and southern regions of Nigeria by verbal autopsy interview. PLoS One. 2017;12(5):e0178129.

13. Kalter HD, Yaroh AG, Maina A, Koffi AK, Bensaid K, Amouzou A, et al. Verbal/social autopsy study helps explain the lack of decrease in neonatal mortality in Niger, 2007-2010. J Glob Health. 2016;6(1):010604. 
14. Kulkarni R, Chauhan S, Shah B, Menon G, Puri C. Investigating the causes of perinatal mortality by verbal autopsy in Maharashtra, India. Indian Journal of Community Medicine. 2007;32(4):259-63.

15. Nonyane BAS, Kazmi N, Koffi AK, Begum N, Ahmed S, Baqui AH, et al. Factors associated with delay in care-seeking for fatal neonatal illness in the Sylhet district of Bangladesh: results from a verbal and social autopsy study. Journal of Global Health. 2016;6(1):010605.

16. Koffi AK, Mleme T, Nsona H, Banda B, Amouzou A, Kalter HD. Social autopsy of neonatal mortality suggests needed improvements in maternal and neonatal interventions in Balaka and Salima districts of Malawi. Journal of Global Health. 2015;5(1):010416.

17. Ashraf RN, Jalil F, Zaman S, Karlberg J, Khan SR, Lindblad BS, et al. Breast feeding and protection against neonatal sepsis in a high risk population. Arch Dis Child. 1991;66(4):488-90.

18. Debes AK, Kohli A, Walker N, Edmond K, Mullany LC. Time to initiation of breastfeeding and neonatal mortality and morbidity: a systematic review. BMC Public Health. 2013;13.

19. United Nations. Transforming our World: the 2030 Agenda for Sustainable Development. New York: UN General Assembly; 2015. Available from:

http://www.un.org/en/development/desa/population/migration/generalassembly/docs/globalcomp act/A_RES 70 1_E.pdf

20. Niermeyer, S. Global gains after Helping Babies Breathe. Acta Paediatr. 2017;106(10):1550-1.

21. Gon G, Ali SM, Towriss C, Kahabuka C, Ali AO, Cavil S, et al. Unpacking the enabling factors for hand, cord and birth-surface hygiene in Zanzibar maternity units. Health Policy Plan. 2017;32(8):1220-8 